\title{
Evaluación del manejo de dos tipos de suelos del departamento del Cesar a través de áreas homogéneas de tierra
}

Luis Díaz', Neill Hamburger²

\section{Resumen}

El departamento del Cesar, a través del Instituto Geográfico Agustín Codazzi (IGAC), presenta mal manejo de los suelos en un $56 \%$, pero esta información es a nivel exploratorio (escala 1:100000) y se desea verificar por medio de un estudio de detalle (escala 1:25000) a través de Áreas Homogéneas de Tierra (AHT). Por ello, este informe tiene como objetivo evaluar el manejo de dos tipos de suelos del departamento del Cesar intervenidos por el ser humano, a través del cálculo de potencial de suelo de la metodología de áreas homogéneas de tierra. La primera área que se estudiará será la utilizada para la minería (SUM) y, la segunda, la de los suelos utilizados en ganadería (SUG). Los resultados arrojaron que lo SUM se categorizan como plintosoles, gracias a los óxidos de hierro presentes en ellos y por el material agregado al suelo como la plintita, mientras que los SUG se caracterizan por ser suelos acrisoles, debido al alto contenido de arcilla y bioturbación del perfil del suelo. Además, los SUM tienen un potencial de 28 , considerado para suelos malos, pero que con controles especiales se puede hacer agricultura y ganadería, mientras que los SUG son moderadamente buenos, aptos para agricultura y no ganadería. De modo que, tras el análisis de áreas homogéneas de tierras, se encontró que los suelos tienen una unidad cartográfica de suelo (UCS) de 10 CSc-28 y 04 CSc 68, respectivamente, para SUM y SUG, indicando que el suelo se encuentra mal utilizados en un $53 \%$.

Palabras clave: plintosol, acrisol, Unidad Cartográfica de Suelos, minería, ganadería.

\footnotetext{
IIngeniero de minas, docente de la Maestría en Ciencias Ambientales de la Universidad Popular del Cesar.

${ }^{2}$ Estudiante de Maestría en Ciencias Ambientales de la Universidad Popular del Cesar. Correo: neillhamburger@unicesar.edu.co
} 


\section{Introducción}

"La génesis de un suelo o edafogénesis consiste en un conjunto de procesos progresivos por los cuales un material orgánico" (Porta, 2004), "posiblemente isotrópicos se transforma en un suelo con uno o más horizontes" (The Royal Society, 2010).

Los estudios sobre génesis de suelos refieren a las investigaciones realizadas para explicar el origen y modo de como se ha formado un suelo, a partir de un material originario, que puede ser una roca in situ, un material transportado o un suelo anterior (Porta, 2004).

"La apariencia externa, los componentes, la organización y la correlación entre las propiedades físicoquímicas del suelo en un momento dado permiten identificar la evolución de un suelo" (Burbano Orjuela, 2010).

Colombia, a través del IGAC, ha adoptado una estrategia para valorar la evolución, categorización y manejo de suelos a través de potenciales del suelo. Esta estrategia la han denominado "Áreas Homogéneas de Tierras" (IGAC, 2012), la cual considera los principales factores formadores de suelo, clima, uso actual del suelo, relieve, regímenes de temperatura, etc. Además de ello, correlaciona las propiedades físico químicas de los suelos para determinar su potencial de uso. Este estudio se ha elaborado a una escala 1:500 000, es decir, solo se ha hecho de forma exploratoria (IGAC, 2012), por lo que requiere que se haga con mayor precisión a una escala 1:25000, es decir, que sea de detalle. Hasta ahora solo han avanzado en esta nueva escala en un 7,4\% y resta por verificar un 96,6\% (IGAC, 2012).

El departamento del Cesar, tras el estudio anterior de áreas homogéneas de tierra, encontró que el departamento en un $56 \%$ está siendo mal utilizando. De verificar en un $96,6 \%$ el problema acrecentará o disminuirá, lo que dificulta determinar la evolución, estado de madurez del suelo, su potencial y, menor aún, la claridad en la correlación de sus propiedades físicoquímicas al ser utilizado. Por ello, este informe tiene como objetivo evaluar el manejo de dos tipos de suelos del departamento del Cesar, a través de áreas homogéneas de tierra. 


\section{Materiales y métodos}

\section{Área de estudio}

El Departamento del Cesar se encuentra dividido en seis regiones naturales, que representan igual número de zonas ecológicas: Sierra Nevada de Santa Marta, Serranía del Perijá, Complejo cenagoso de Zapatosa, Valle del río Cesar, Valle del río Ariguaní y Valle del Magdalena. El área de estudio estará en el valle del río Cesar, ocupa la parte central del Departamento y hace parte de la Llanura del Caribe, son tierras planas u onduladas con altitudes entre los 50 y 200 m.s.n.m. cubiertas de pastizales y bosque claro. En este sector se encuentran las cabeceras municipales de Valledupar, Agustín Codazzi, La Paz, San Diego, Curumaní, Chiriguaná. Con precipitaciones en torno a los $1000 \mathrm{~mm}$. A nivel térmico presenta temperaturas superiores a los $28^{\circ} \mathrm{C}$ de media anual. (CDT-G, 2011)

Los principales renglones económicos del Cesar son el agropecuario del que deriva un $30 \%$ de sus ingresos, el de servicios con el $35 \%$ y la minería con el $27 \%$ de los mismos (una parte de la minería es explotada de manera ilegal). La ganadería vacuna ocupa un lugar de primer orden, con una población estimada en 1.513.149 cabezas. (ACP, 2010)

El cultivo y procesamiento de algodón, las palmas oleaginosas y otros productos agrícolas alcanzan altos niveles de tecnificación y desarrollo. Cesar es el primer productor nacional semilla de palma africana y el segundo de arroz. La elaboración de productos lácteos y de grasas de aceites figuran igualmente entre sus principales industrias. También existe una amplia extensión de cultivos de frutas. (FIDA, 2010)

En la última década el departamento del Cesar ha tomado un incremento en su economía debido a la explotación de minas de carbón a cielo abierto liderada por la empresa multinacional Drummond, y otros más, principalmente en el municipio de El Paso y La Jagua de Ibirico, siendo últimamente afianzada por el hallazgo de grandes yacimientos en el Sitio Conocido como el Descanso. A partir de 2004, el Cesar se convirtió en el primer productor nacional de Carbón. (Minminas, 2012) 


\section{Muestreo}

El muestreo se hizo en dos áreas expuestas a actividades antrópicas por minería y ganadería. Las muestras de suelo utilizadas en la minería (SUM) se tomaron en el trayecto hacia el municipio de La Loma, Cesar, en las coordenadas N9³7'50,3”' W73³2'51,7’, y las muestras de suelo utilizadas en la ganadería (SUG) hacia la vereda Estados Unidos del municipio La Jagua de Ibirico se encuentra en las coordenadas N9³8'56,7' W73¹6'34,3”. Se elaboró una alicata de $1 \mathrm{~m}$ de profundidad y se tomó un $1 \mathrm{~kg}$ de muestra de suelo por cada horizonte para análisis en el laboratorio. Se registró profundidad efectiva, de zona de bioturbación, textura, plasticidad, consistencia, vegetación, clima, temperatura del suelo, temperatura ambiental, color de suelo en húmedo y seco con tablas Munsell; se identificó la presencia de materia orgánica con peróxido de hidrógeno grado analítico, $\mathrm{pH}$ por tira de colores y calcio, con una solución de $\mathrm{HCl}$ al $10 \%$.

\section{Caracterización fisicoquímica}

"Para caracterizar el pH y la conductividad eléctrica fueron medidos en proporción suelo-agua 1:1 y 1:5 respec- tivamente" (IGAC, 2008). "El carbono orgánico del suelo (COS) fue determinado por el método de oxidación húmeda con dicromato potásico conocido como método Walkley-Black" (Mingorance, 2007). "El fosforo se cuantificó por el método Olsen" (IGAC, 2008). "La extracción del fosforo se hizo con solución de bicarbonato de sodio $0,5 \mathrm{~N}$ a pH 8,5. La capacidad de intercambio catiónico según el IGAC" (IGAC, 2008). "La densidad real y aparente por la ntc 5167, la textura por el método Boyoucus, el color del suelo por notación Munsell" (Jaramillo, 2000), "tamaño de poros, porosidad y humedad del suelo según Jarami1lo" (Jaramillo, 2000).

\section{Análisis de áreas homogéneas de tierras}

Para evaluar el manejo de uso del suelo se calculó el potencial del suelo, utilizando la metodología planteada por el IGAC en la IV Reunión Anual del Comité Permanente Sobre el Catastro en Iberoamérica CPCI y de acuerdo con el taller de Agrología liderado por el IGAC regional del Cesar (German, 2017). La cual consiste en valorar las características fisicoquímicas del suelo en campo y laboratorio, permitiendo dar una apreciación del valor potencial de uso (tabla 1). 
Tabla 1. Apreciación de la calidad de uso del suelo (IGAC, 2017)
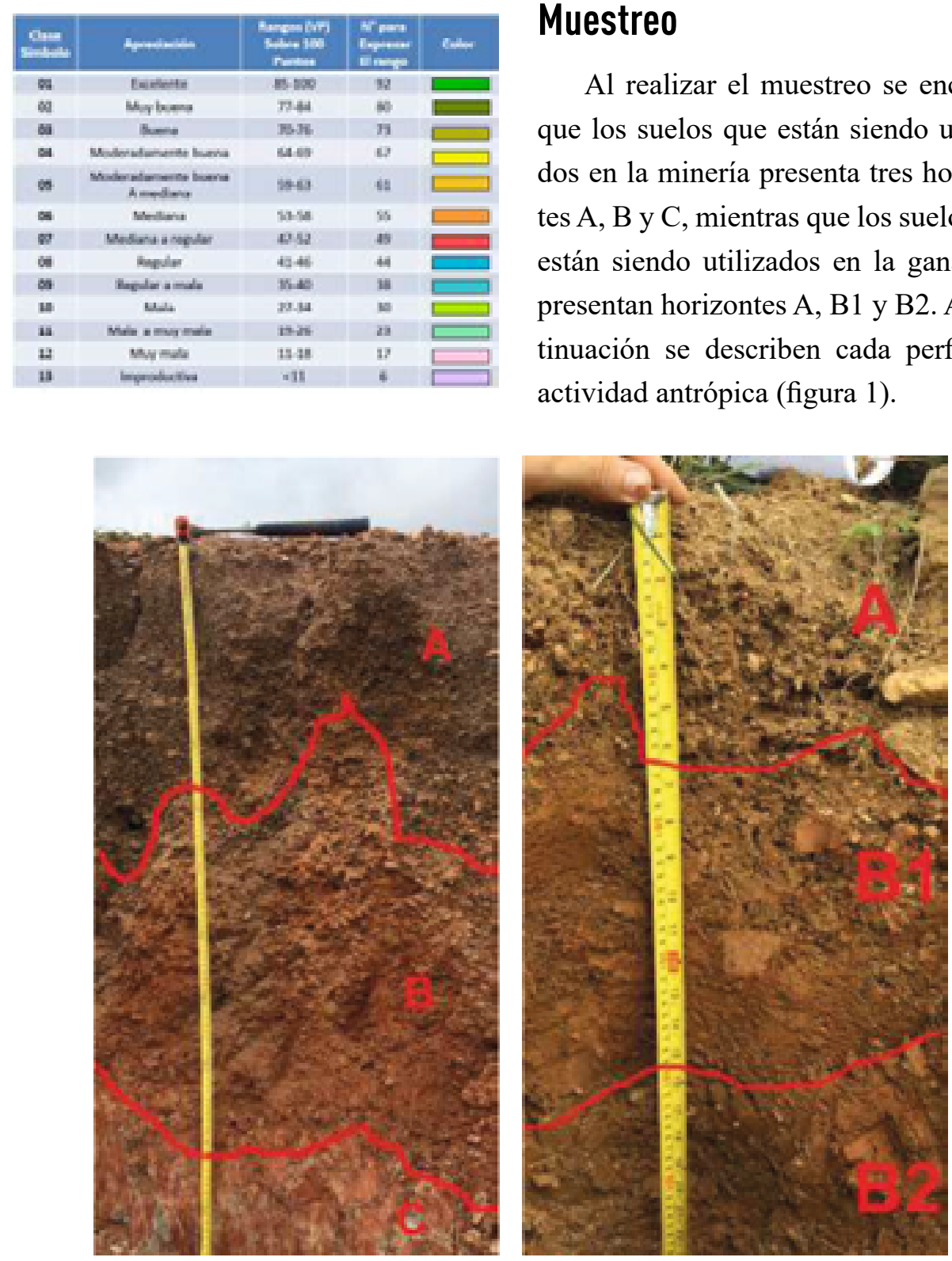

Figura 1. Perfil de suelo por actividades antrópicas. Derecha SUM, Izquierda. SUG

\section{Resultados}

\section{Muestreo}

Al realizar el muestreo se encontró que los suelos que están siendo utilizados en la minería presenta tres horizontes A, B y C, mientras que los suelos que están siendo utilizados en la ganadería presentan horizontes A, B1 y B2. A continuación se describen cada perfil por actividad antrópica (figura 1).

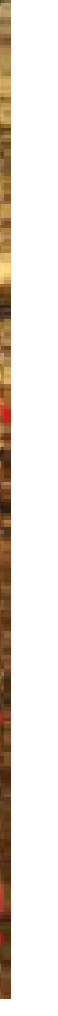

(1) 
Descripción de suelos utilizados en la minería (SUM)

El horizonte A tiene una profundidad de 40-50 cm de material granular, con una melanización de color 2,5YR $3 / 1$. Una textura arenosa, con presencia de bioturbación por raíces. Horizonte B, de $46-60 \mathrm{~cm}$ material granular con color de suelo 2,5 YR 4/8, textura franca arcillo arenosa, con presencia de bioturbación de raíces hasta los primeros $25 \mathrm{~cm}$ de iniciado el horizonte. El horizonte C se presenta a mayor profundidad; de un metro, con moteados de hierros color característico 2,5 YR 5/8 y textura arcillosa. Con una pendiente del $10 \%$, la zona es ondulada (tabla 2).

En cuanto a sus características fisicoquímicas, el suelo en sus tres horizontes tiene condiciones bajas de $\mathrm{pH}, \mathrm{CE}$, CIC y Fósforo, se observa en la tabla 2, a excepción del carbono orgánico que presenta de forma elevada en todo el perfil y menor a medida que avanza en profundidad, pero que esta podría estar relacionado con el carbón presente de la zona. En campo se le adiciono peróxido de hidrógeno al suelo y dio negativo para carbono orgánico. Ahora, la humedad en el horizonte $\mathrm{C}$ presenta una mayor retención de agua, debido a la composición de arcilla que posee. 
Tabla 2. Ficha de muestreo en campo SUM

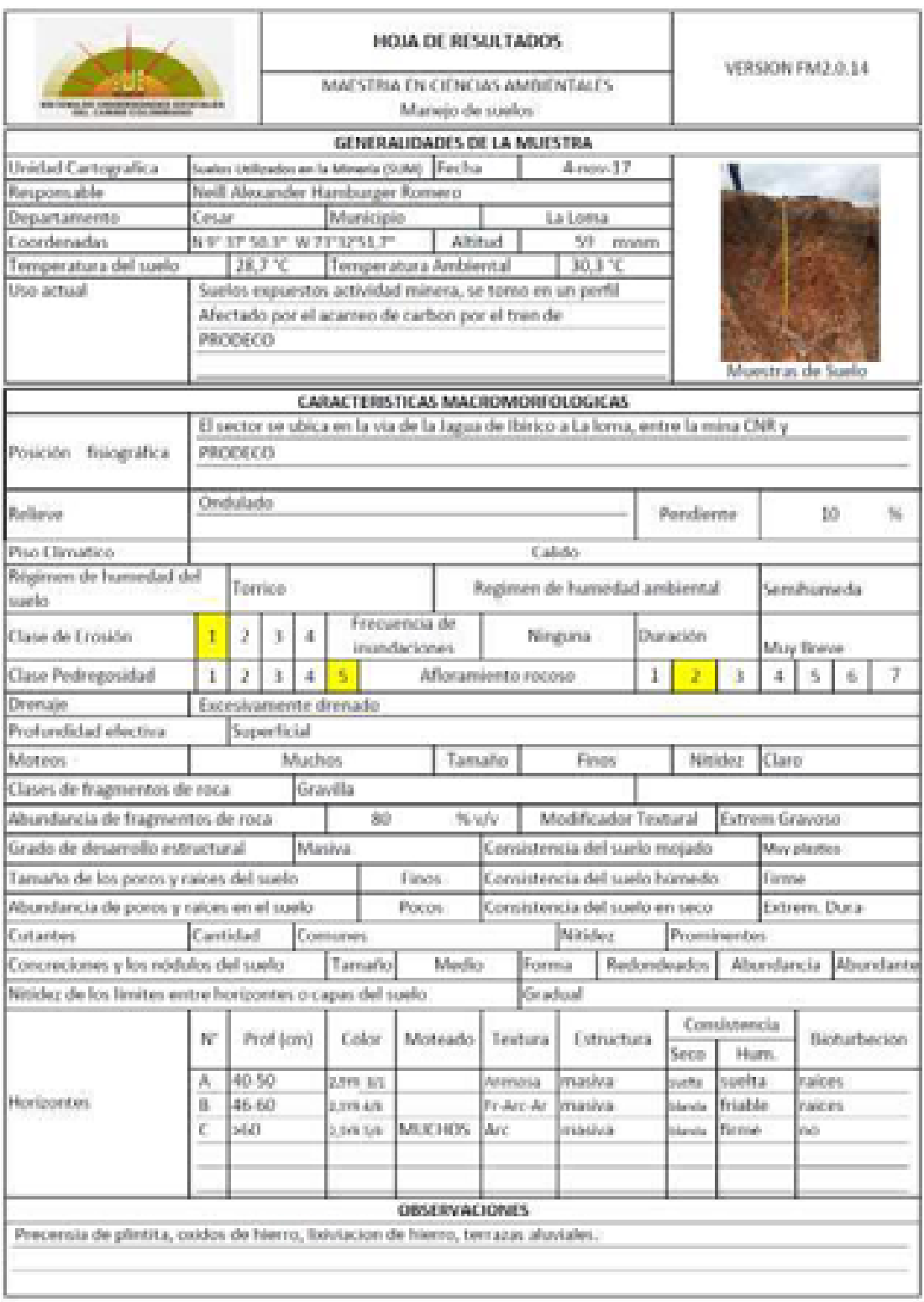


Tabla 3. Caracterización fisicoquímica de horizontes de suelos utilizados en la minería

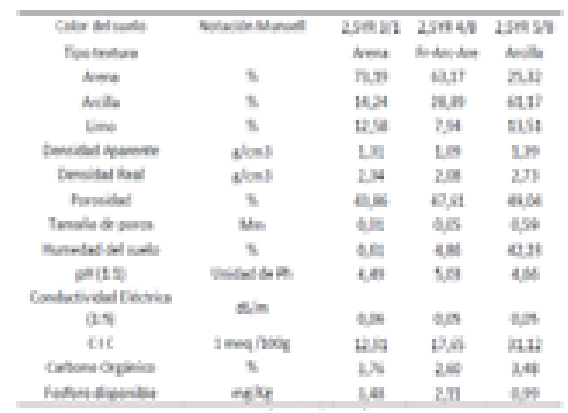

Fuente: elaboración propia.

Este mismo perfil está sometido a la interacción de las siguientes especies vegetales: palma de vino, pasto Brysanta, Byrsonima Sp (Peralejo), Mimosa Sp, Fabaseas Sp. Además, presenta terrazas aluviales, erosión grado dos, elementos consolidados de plintita, fragmentos de cuarzo y lixiviación de hierro en el horizonte $\mathrm{C}$.
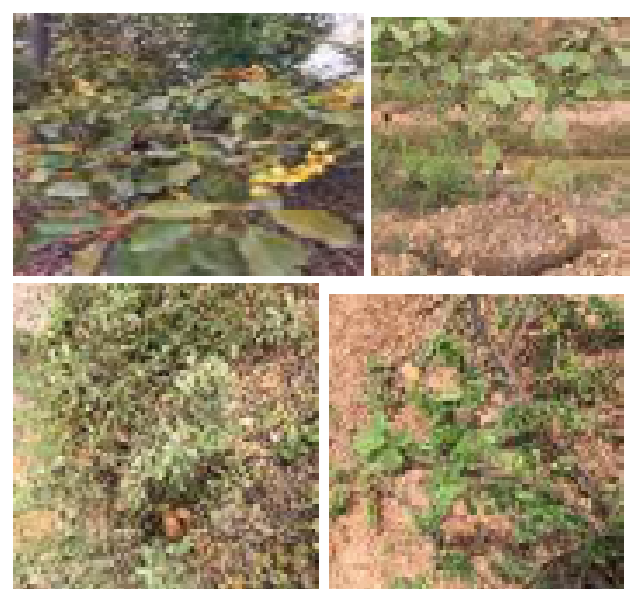

Figura 2. Vegetación del lugar
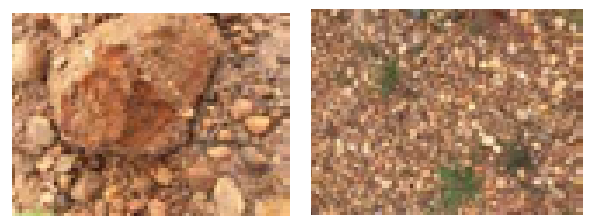

Figura 3. Características del paisaje. Plintita, fragmentos de cuarzo, lixiviación de hierro

Por toda la descripción anterior, se puede concluir que el suelo es un plintosol, debido a que en la WRB (2006) describen el plintosol como suelos que presentan plintita, petroplintita o pisolitos, tras un proceso de meteorizado de rocas acídicas, con presencia abundante de Fe, asociado con áreas llanas suavemente inclinadas con agua freática fluctuante y en cuanto al des arrollo del perfil. "La petroplintita puede romperse en agregados irregulares o gravas, que pueden ser transportadas para formar depósitos coluviales o aluviales. Como se detecta y evidencia en el muestreo de las dos zonas" (WRB, 2006) Según la WRB (2006), el manejo y uso que se le debe dar a esta clase de suelo es agrícola y forestales (por ejemplo, caco, castañas de cajú, etc.); con técnicas de manejo del suelo y agua se puede utilizar agricultura urbana y preurbana. Así mismo, se puede utilizar en minería debido a la plintita y petroplintita presentes en el suelo. "La plintita es un material esencial en la 
fabricación de ladrillos y la petroplintita en material de grava para construcciones" (WRB, 2006).

Descripción de Suelos Utilizados en la Ganadería (SUG). El horizonte A tiene una profundidad de $20-30 \mathrm{~cm}$, con una melanización de color 5 YR 4/3 y una textura arcilloarenosa con presencia de bioturbación por raíces e insectos. El horizonte $\mathrm{B} 1$ tiene una profundidad de 30-60 cm, con color de suelo 5 YR 5/6, textura arcillosa, con presencia de bioturbación de raíces e insectos. El horizonte B2 se presenta a mayor profundidad, de $60 \mathrm{~cm}$, con moteados de hierros color característico 5YR 6/6 y textura arcillosa. Con una pendiente del 10\%, la zona es ondulada (tabla 4).

En cuanto a sus características fisicoquímicas, el suelo en sus tres horizontes tiene condiciones bajas de humedad,
pH, CE y CIC (tabla 5), a excepción del carbono orgánico que se presenta de forma elevada en todo el perfil y menor a medida que avanza en profundidad. En campo se le adicionó peróxido de hidrógeno al suelo y dio positiva para carbono orgánico. A medida que aumenta la profundidad aumenta el fósforo, pero esto podría estar relacionado con la lixiviación de fósforo de los perfiles. La porosidad es aceptable para el horizonte A y disminuye debido al contenido de arcilla. Este mismo perfil está sometido a la interacción de las siguientes especies vegetales: pasto Brysanta, Byrsonima Sp (peralejo), Fabaseas Sp. Además, presenta terrazas aluviales, presenta erosión tipo 1, elementos consolidados, lixiviación de hierro en el horizonte $\mathrm{C}$ y el terreno presenta bastante pedregosidad, cantos rodados (figuras 4 y 5). 
Tabla 4. Ficha de muestreo en campo SUG

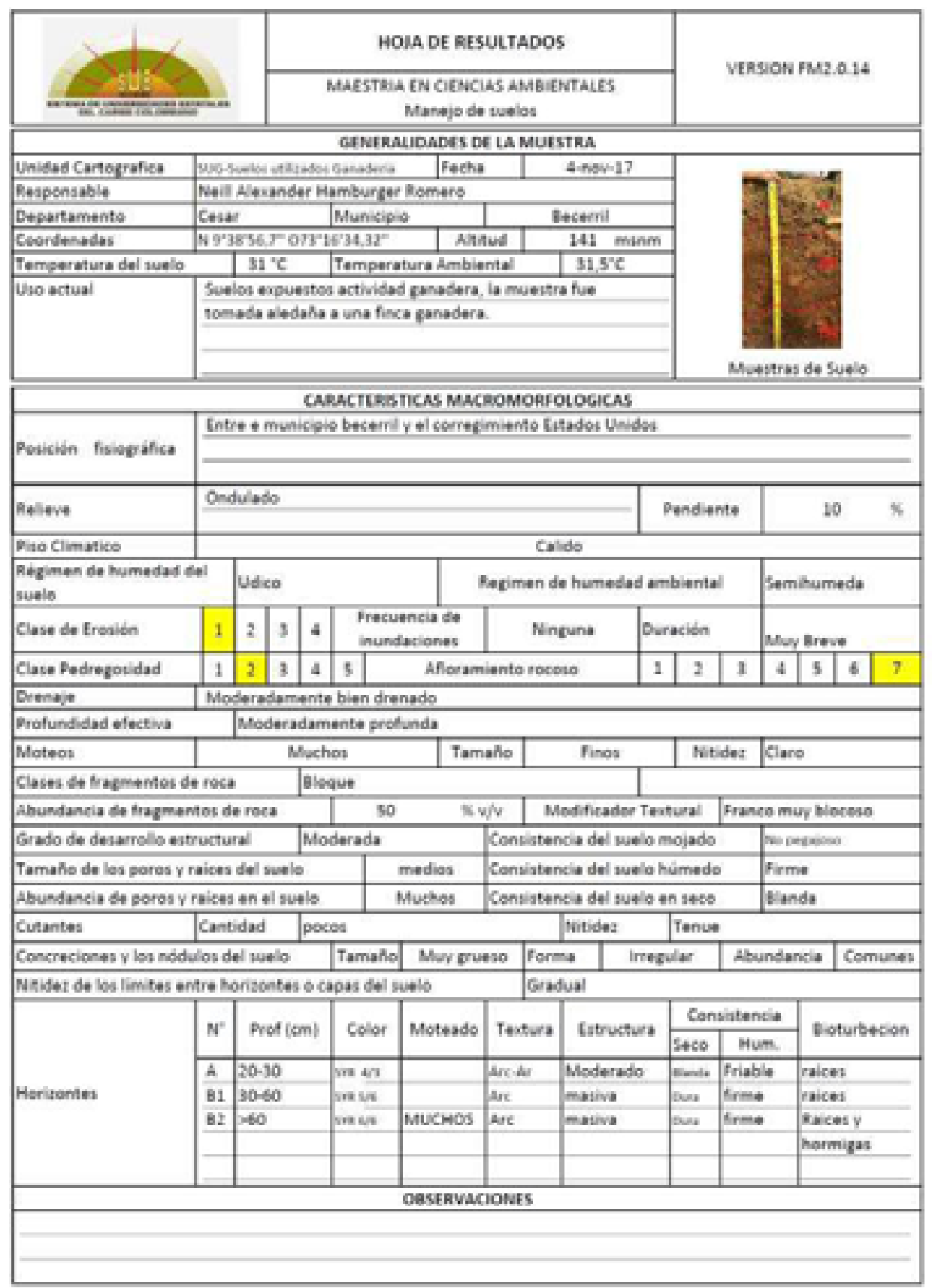


Tabla 5. Caracterización fisicoquímica de horizontes de suelos utilizados en la ganadería

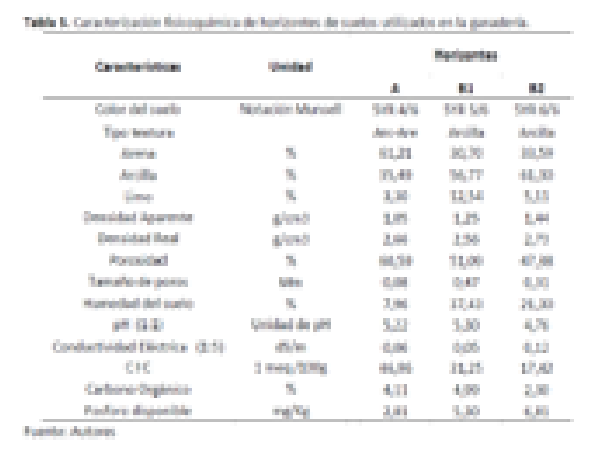

Fuente: elaboración propia.
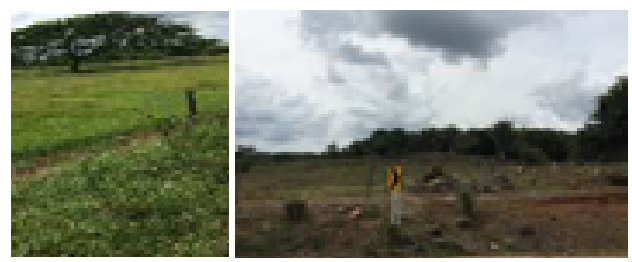

Figura 4. Vegetación del lugar

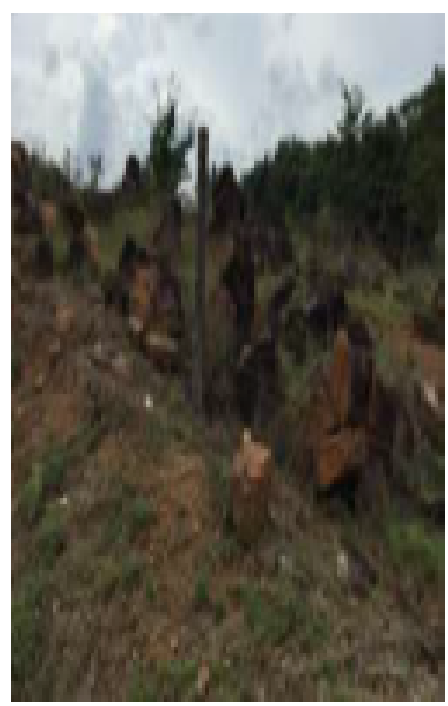

Figura 5. Características del paisaje
Por toda la descripción anterior, se puede concluir que el suelo es un acrisol, debido a que en la WRB (2006) "describe que son suelos que tienen mayor contenido de arcilla en el subsuelo que en el suelo superficial como resultado de procesos pedogenéticos (especialmente migración de arcilla) que llevan a un horizonte árgico en el subsuelo". Los acrisoles tienen en determinadas profundidades una baja saturación con bases y arcillas de baja actividad. Muchos acrisoles correlacionan con Red Yellow Podzolic Soils, Argissolos).

En cuanto al desarrollo del perfil es diferenciado pedogenética del contenido de arcilla con un bajo contenido en el suelo superficial y mayor contenido en el subsuelo; lixiviación de cationes básicos debido al ambiente húmedo y avanzado grado de meteorización. (WRB, 2006)

Según la WRB (2006)

el manejo y uso que se le debe dar a los acrisoles es la preservación del suelo superficial, con el fin de conservar la materia orgánica y prevenir la erosión. Ya que el desmonte mecánico de la superficie produce tierras que son muy estériles cuando 
la concentración de Al del subsuelo alcanza niveles tóxicos. Se requieren sistemas de cultivo adaptados con fertilización completa y manejo cuidadoso si se va a practicar agricultura sedentaria en acrisoles. La agricultura de cortar y quemar ampliamente utilizada (agricultura nómade, shifting cultivation) puede parecer primitivo, pero es una forma de uso de la tierra bien adaptada, desarrollada durante siglos de prueba y error. Si los períodos de ocupación son cortos (solo uno o unos pocos años) y seguidos por un período de regeneración suficientemente largo (hasta varias décadas), este sistema hace un buen uso de los recursos limitados de los acrisoles. Se recomienda la agroforestación como una alternativa que protege al suelo frente a la agricultura nómade para alcanzar altos rendimientos sin requerir insumos costosos. (WRB, 2006)

La agricultura de bajos insumos en acrisoles no es muy gratificante. Los cultivos de cosecha no demandantes, tolerantes a acidez como el ananá, castaña de cajú, té y caucho pueden cultivarse con algún éxito. Áreas crecientes de acrisoles se plantan con palma aceitera (p. ej., en Malasia y en Sumatra). La mayoría de las raíces de los árboles se concentran en el horizonte superficial humífero con sólo unas pocas raíces principales se extienden hacia abajo en el subsuelo. En Sudamérica los acrisoles también se encuentran bajo sabana. Los acrisoles son apropiados para la producción de cultivos de secano o irrigados sólo después de encalado y fertilización completa. La rotación de cultivos anuales con pasturas mejoradas mantiene el contenido de materia orgánica (WRB, 2006).

\section{Análisis de áreas homogéneas}

El análisis de áreas homogéneas de tierra para los dos suelos intervenidos por actividades antrópicas arrojo que el SUM se categorizó (tabla 6) como 10 CSc-28 con un potencial de 28 equivalente para uso en la agricultura con controles, la ganadería y, finalmente, la minería, en concordancia con WRB (2006). El uso y manipulación actual del suelo permite asegurar que se encuentran mal manejado. Por su lado, el SUG se categorizó (tabla 7) como 04 CSc-67, con un potencial de 67 equivalente para agricultura y ganadería; en concordancia con la 
WRB (2006) también se encuentra mal manejado este suelo (German, 2017).

Ahora el IDEAM (2014) presenta estos suelos con un potencial de 28 para SUM y de 68 para SUG, lo que deja ver que hay un coeficiente de variación del $0,05 \%$ e implica que se verificó un área equivalente al $3 \%$ del territorio del departamento del Cesar, evidenciando así que los suelos están siendo mal manejados en un $53 \%$ (German, 2017). 
Tabla 6. Análisis de áreas homogéneas de tierra para SUM

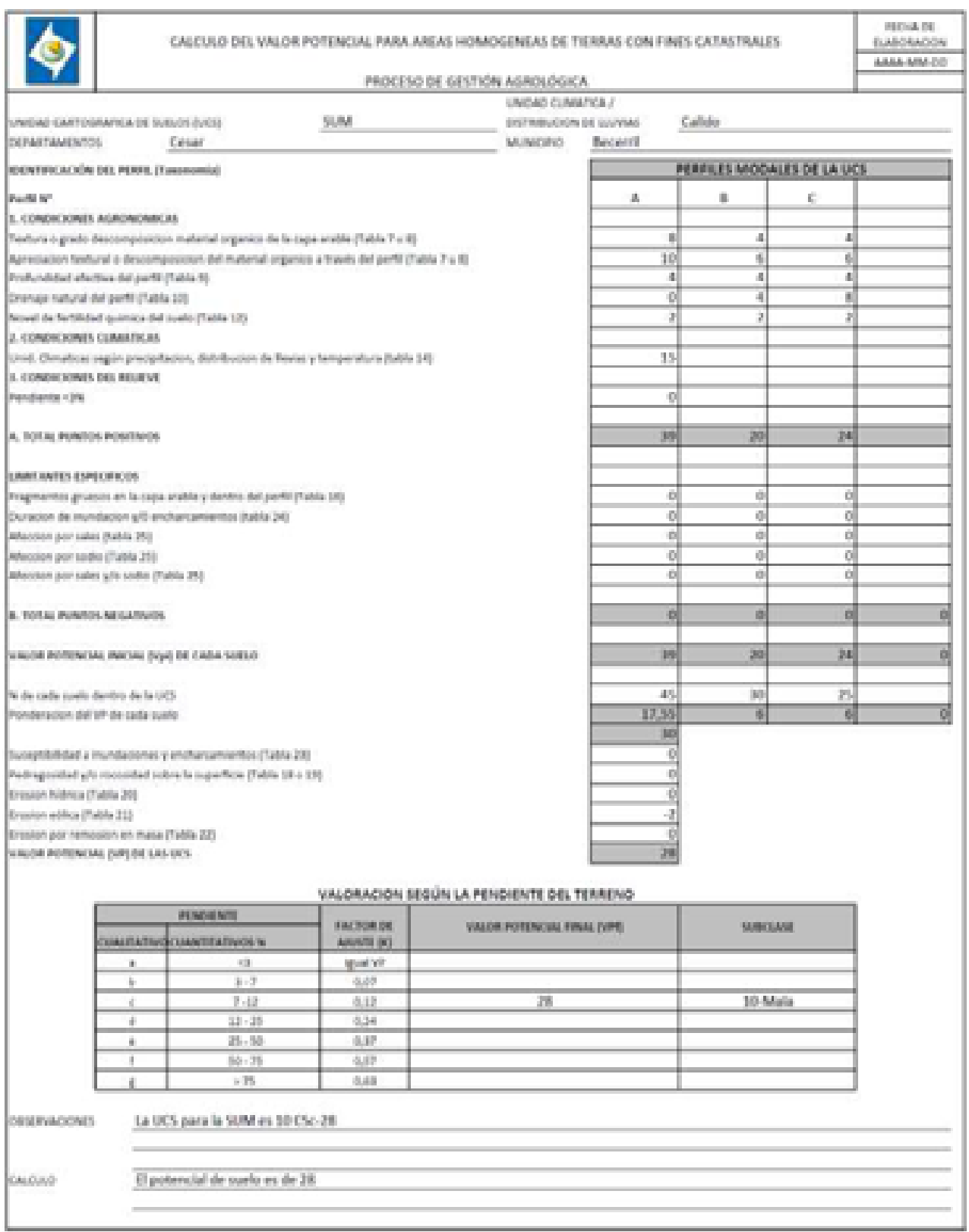


Tabla 7. Análisis de áreas homogéneas de tierra para SUG

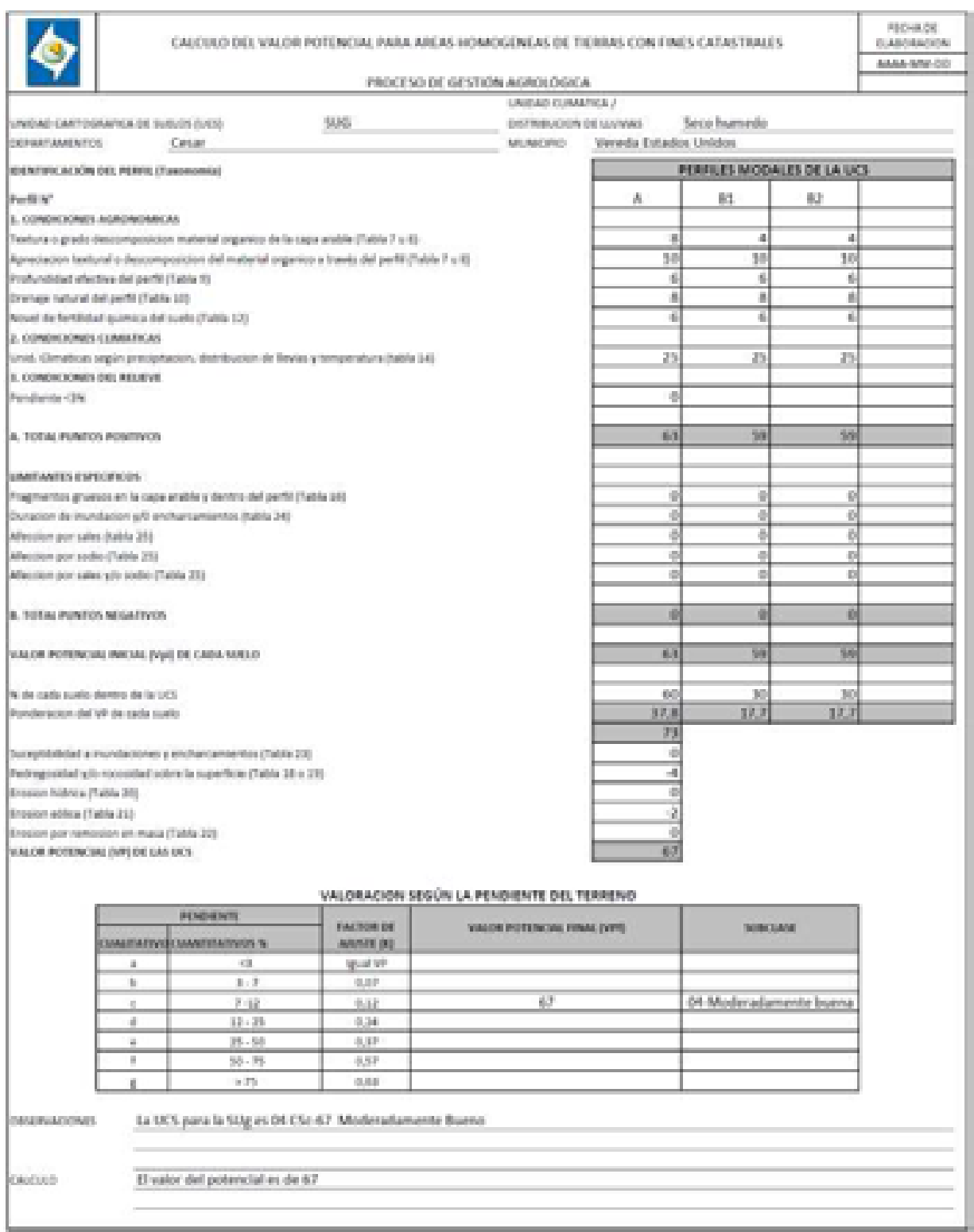




\section{Conclusiones}

Los suelos SUM se categorizan como plintosoles gracias a los óxidos de hierro presentes en ellos y por el material agregado al suelo como la plintita, mientras que los SIG se caracterizan por ser suelos acrisoles debido al alto contenido de arcilla y bioturbación del perfil de suelo.

Los suelos utilizados en minería tienen un potencial de 28 suelos considerados malos, que con controles especiales se puede hacer agricultura y ganadería, mientras que los suelos utilizados por ganadería son moderadamente buenos aptos para agricultura y no ganadería.

Tras el análisis de áreas homogéneas de tierras se encontró que los suelos tienen una unidad cartográfica de suelo (UCS) de 10 CSc-28 y 04 CSc 68, respectivamente, para SUM y SUG. Indicando que el suelo se encuentra mal utilizados en un $53 \%$.

\section{Referencias}

ACP. (2010). Sector minero y petrolero en Colombia. Asociación Colombiana del Petróleo-ACP: Colombia.

Burbano Orjuela, H. (2010). El suelo al servicio de la sociedad y su rol en el contexto de los Cambios Globales. Revista Tendencias, $X I(2)$, 53-62.
CDT-G. (2011). Plan estratégico para el centro de investigación y desarrollo tecnológico para la innovación del CDT del Cesar. Centro de investigación y Desarrollo Tecnológico Ganadero, 1-352.

FAO. (2007). Land evaluation. FAO Land and Water Discussion Paper 6.

FAO. (2016). FAO.org. Recuperado el 24 de 11 de 2016, de http://www.fao.org/soilsportal/

levantamiento-de-suelos/propiedades-del-suelo/propiedades-quimicas/es/

FIDA. (2010). Fondo Internacional de Desarrollo Agrícola. Desertificación, 4.

German, D. (2017). Taller de Áreas Homogéneas de Tierra. IGAC-Cesar.

IGAC. (2012). Estudio de los conflictos de uso del territorio colombiano escala .1:100.000. Instituto Geográfico Agustín Codazzi. Bogotá. Imprenta Nacional de Colombia.

Jaramillo, (2000). Ciencia del suelo. Medellín.

Mingorance, M. E.-G. (2007). Guidelines for improving organic carbon recovery by the wet oxidation method. Chemosphere, 68 , 409-413.

Minminas. (2012). ABC Minero. Documento de apoyo para el proceso de consulta previa del "proyecto de ley por medio del cual se reforma el código de minas y se dictan otras disposiciones". Ministerio de Minas y Energía. Colombia.

Porta. (2004). Edafogenesis. España.

SIAC. (2012). Sistema de Información Ambiental en Colombia. Recuperado el 23 de septiembre de 2012, de http://www. siac.gov.co/contenido/contenido.aspx?co$n I D=1000 \&$ cat $I D=316$ 
SIMCO. (2014). Sistema de Información Minero Colombiano.

The Royal Society. (22 de agosto del 2010).

"Geoengineering the climate: science, governance and uncertainty". Recuperado de http://royalsociety.org/Geoengineering-the-climate

WRB. (2006). Referencia de Suelos. FAO. 